\title{
Evaluation of Focused Ion Beam for Shave-off Depth Profiling
}

\author{
M. Fujii, ${ }^{1 *}$ T. Imamura, ${ }^{1}$ M. Nojima ${ }^{2}$ and M. Owari ${ }^{1,3}$ \\ ${ }^{I}$ IIS, The University of Tokyo, 4-6-1 Komaba, Meguro-ku, Tokyo 153-8505, Japan \\ ${ }^{2}$ RIST, Tokyo University of Science, 2641 Yamazaki, Noda, Chiba 278-8510, Japan \\ ${ }^{3}$ ESC, The University of Tokyo, 7-3-1 Hongo, Bunkyo-ku, Tokyo 113-0033, Japan \\ *m-fujii@iis.u-tokyo.ac.jp
}

(Received : October 3, 2010; Accepted : December 10, 2010)

\begin{abstract}
Shave-off depth profiling is one of the powerful methods that can be applied to the analysis of pin point and small region devices. However, acquired shave-off depth profile is affected by a long tail of the FIB because shave-off scanning mode has the distinctive position of the primary ion beam against the sample. In this study, we evaluated the influence of the long tail of the FIB in detail by the simulation method we newly proposed. The evaluation was carried out from the point of view of mainly two factors. One is thickness of the protection film. The other is the scanning speed toward the depth direction. In addition, we newly proposed the notion of 'shave-off sputtered atom yield', the important factor of shave-off depth profiling. As a result, it became possible that the determination of optimized experimental condition for the achievement of the ultimate depth resolution.
\end{abstract}

\section{Introduction}

Shave-off depth profiling with nano-beam Secondary Ion Mass Spectrometer (SIMS), our own unique technique, achieves the highly precise depth profiling with nanometer-scaled depth resolution by utilizing a Focused Ion Beam (FIB) micro-machining process to provide the depth profile [1]. This method is a very unique depth profiling for acquiring depth profile by the shave-off scanning mode (Fast horizontal sweeps of an FIB is combined with a very slow vertical sweep). Shave-off depth profiling is one of the powerful methods for analyzing the structured sample: particles, rough surfaces, and/or micro-machined structures. However, the acquired shave-off depth profile is strongly affected by the long tail of an FIB because shave-off scanning mode has the distinctive position of the primary ion beam against the sample. It is widely known that the FIB has a long tail by the cause of spherical aberrations and/or transverse thermal velocity effects [2]. In the shave-off condition, the FIB always keeps to an edge of the sample and shaves off the sample completely from the surface into the depth direction. When the FIB is approaching the sample, the edge of the sample is sputtered by a long tail of the FIB little by little. Thus, the acquired depth profile has a long leading edge of particular shape. This phenomenon makes depth resolution worse.

In our previous study, we reduced the influence of a long tail of the FIB by using deposition film [1,3]. In other previous study, we developed the simulation method that removes the influence of a long tail of the FIB from the acquired shave-off depth profile [4]. This method enabled us to acquire the estimated shave-off depth profile with the depth resolution of about 15 $\mathrm{nm}$. However, the evaluation of sputtering mechanism in the shave-off condition is still insufficient. Thus, we improved the conventional simulation method to evaluate the perspective between the FIB and the sample in the shave-off condition. In this study, we report the new notion of 'shave-off sputtered atom yield' and optimization of experimental condition for the achievement of ultimate depth resolution.

\section{Shave-off Depth Profiling}

It is widely known that the FIB has a long tail outside of the intensity profile by the cause of spherical aberrations and/or transverse thermal effects [2]. In the shave-off depth profiling, the sample is shaved off by not all of the intensity of the beam but the outer part of the beam. When the FIB is approaching the sample, the edge of the sample is sputtered by the long tail of the FIB little by little. Thus, the acquired shave-off depth profile has a long leading edge of particular shape 
that reflects the long tail of the FIB. This phenomenon makes depth resolution worse.

Mainly two methods exist for reducing the influence of the long tail of FIB. One is attaching the protection film in order to avoid the exposure of the long tail to the sample. The other is optimizing the scanning speed in order to make the sample sputtered using up to the center of the beam. The schematic images of these two methods are shown in Figs. 1 and Fig. 2, respectively.

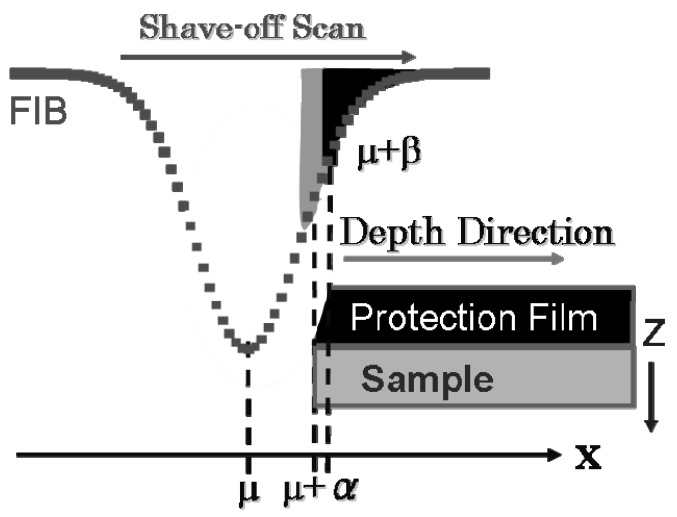

Fig.1. The schematic image of the protection film.
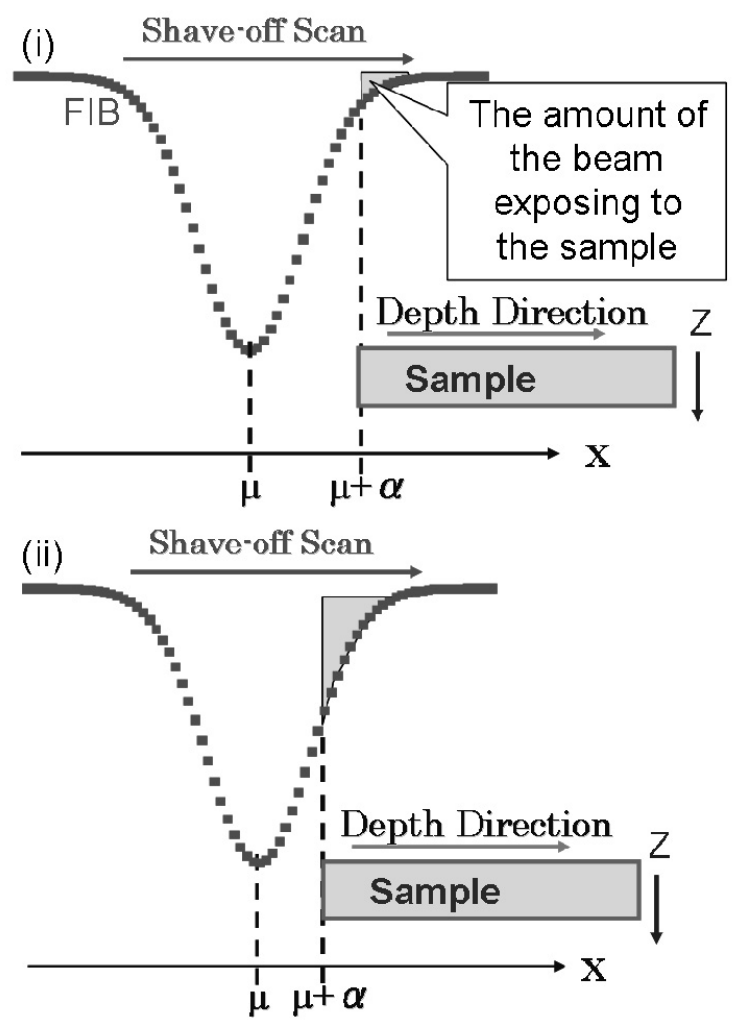

Fig. 2. The schematic image of the changing speed of shave-off scan toward the depth direction.

(i) slow (ii) fast
In order to realize the ultimate depth resolution, we should optimize various experimental conditions, especially the thickness of the protection film and scanning speed toward the depth direction. These two parameters can be determined relying on the 'shave-off sputtered atom yield'. Important point is that 'shave-off sputtered atom yield' is essentially different from the general 'sputtered atom yield', because of the distinctive position of the beam against the sample. 'Shave-off sputtered atom yield' relies on the elemental composition of only $\mathrm{Z}$ direction of the sample. Because the FIB is moving into depth direction at the constant speed and the sample is completely shaved off, the difference of sputtered atom yield of individual atoms composing the sample is needless to consider in the shave-off depth profiling. Thus, only one 'shave-off sputtered atom yield' is determined at every sample even if the sample is compound or mixture.

\section{Simulation}

We assumed that the shave-off depth profile is essentially a convolution of the beam profile with the true elemental distribution [3]. In our previous study, we reported that shave-off depth profile can be simulated more precisely in the case of adopting the convolution of Gaussian and Lorenzian as the intensity profile of FIB compared with in the case of Gaussian. The estimated intensity profile of FIB at shave-off condition was the convolution of Gaussian $30 \mathrm{~nm}$ FWHM with Lorenzian $15 \mathrm{~nm}$ FWHM [4]. However, conventional simulation method excluded the consumption of sample due to the sputtering and just convoluted beam profile with elemental distribution as they were. In order to discuss the effect of protection film, it is important to simulate the extent of sample (including the protection film) sputtered by the FIB. Thus, we improved the conventional simulation method and achieved new method that can consider the consumption of sample due to the sputtering.

In the simulation method we proposed, the signal intensity $I(x)$ can be calculated as:

$$
I(x)=I_{0} \int_{\mu+\alpha}^{\mu+\beta} N(x) G(\mu-x) d x
$$

Here, $\mu$ is the center position of FIB moving into the direction of depth at a constant speed. $x$ is the depth position with its origin at an arbitrary point and $N(x)$ is the concentration of the target atom at depth $x . N(x)$ changes along with being sputtered 
by the beam. $G(x-\mu)$ is the intensity profile of the primary ion beam. We adopted the convolution of Gaussian $30 \mathrm{~nm}$ FWHM with Lorenzian $15 \mathrm{~nm}$ FWHM as the beam profile. $\alpha$ is a parameter indicating a position where the sample is shaved off completely and $\beta$ is a position where the protection film is shaved off completely. $I_{0}$ is proportional constant relies on the ionization yield.

$\alpha$ in the above equation also expresses the amount of the beam exposing to the sample in the unit time. Therefore, we can estimate 'shave-off sputtered atom yield' by using $\alpha$ and sputtered sample volume.

\section{Experimental}

The samples were multi-layer thin film (Al 1 $\mu \mathrm{m} / \mathrm{SiO}_{2} 0.8 \mu \mathrm{m} / \mathrm{Si}$ Substrate and $\mathrm{Al} 0.3 \mu \mathrm{m} / \mathrm{Ti}$ $0.3 \mu \mathrm{m} /$ Si Substrate). They were prepared using FIB micro-machining technique [6]. The size of the thinned samples was $6.5 \mu \mathrm{m}$ in width, $1 \mu \mathrm{m}$ in thickness and $6 \mu \mathrm{m}$ in height. A protection film of carbon of about $0.3 \mu \mathrm{m}$ thickness was deposited on the sidewall of the sample in order to protect the sample from damage by the long tail of FIB during the shave-off depth profiling.

Different shave-off depth profiles were acquired by changing the experimental condition of scanning speed toward the depth direction. The primary ions of this apparatus were field-emitted $\mathrm{Ga}^{+}$with $30 \mathrm{keV}$ energy and $35 \mathrm{pA}$ beam current. The mass analyzer was a modified Mattaugh-Herzog type that is originally equipped with a spark source mass spectrograph (JEOL JMS01BM).

Depth resolution of each acquired depth profile and 'shave-off sputtered atom yield' of Al, $\mathrm{Ti}$ and Si were estimated.

\section{Results and Discussions}

\subsection{Simulation}

Figure 3 shows the relationship between depth resolution and thickness of protection film. The simulation conditions were sample thickness $1 \mu \mathrm{m}$ and scanning speed $7.8 \mathrm{~nm} / \mathrm{s}$ and assumed sample was Al. This result clearly indicates that the thicker the protection film is attached, the higher the depth resolution changes.

Then, Fig. 4 shows the relationship between depth resolution and the scanning speed of FIB toward depth direction. The simulation conditions were the sample thickness $1 \mu \mathrm{m}$, protection film thickness $0.3 \mu \mathrm{m}$ and assumed sample was Al. This result suggests that the faster the speed of shave-off scan becomes, the smaller the influence of long tail of FIB changes. This is because the sample was sputtered using up to the center of the beam in the case of the fast scan. The highest depth resolution is achieved at scanning speed about $12 \mathrm{~nm} / \mathrm{s}$ in this simulation condition. In the condition of more than $12 \mathrm{~nm} / \mathrm{s}$ scanning speed, the sample is sputtered not completely and remains after shave-off profiling. These conditions are difficult to realize in the practical experiment.

From these results, it appeared that the optimized conditions can be determined using this new simulation method for every measurement. For example, when the thickness of protection film is about $0.5 \mu \mathrm{m}$, the thickness of the $\mathrm{Al}$ sample (excluding protection film) is $1 \mu \mathrm{m}$ and scanning speed $12 \mathrm{~nm} / \mathrm{s}$, the ultimate depth resolution of $13 \mathrm{~nm}$ is achieved.

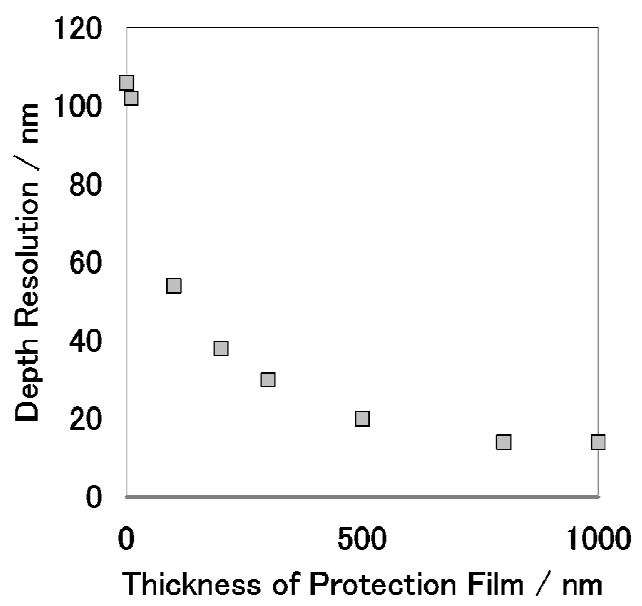

Fig. 3. The relationship between depth resolution and the thickness of protection film.

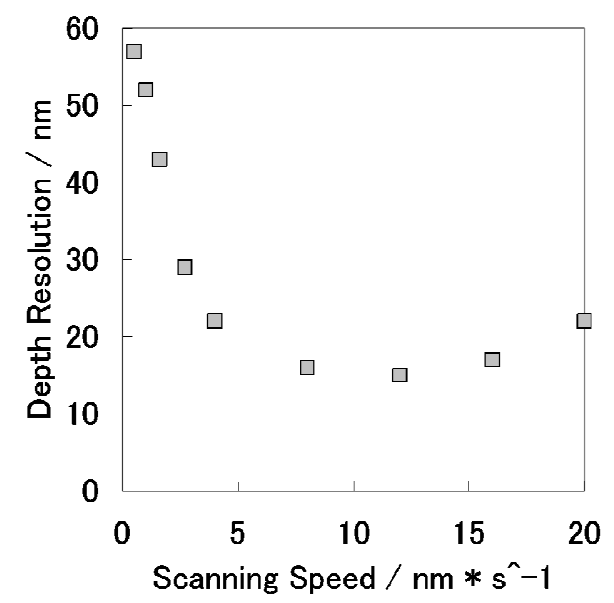

Fig. 4. The relationship between depth resolution and the scanning speed toward depth direction. 


\subsection{Experimental}

Figure 5 shows the leading edge of acquired shave-off depth profiles of $\mathrm{Al}^{+}$in $\mathrm{Al}, \mathrm{Ti}^{+}$in $\mathrm{Ti}, \mathrm{Si}^{+}$ in $\mathrm{Si}$ and $\mathrm{Si}^{+}$in $\mathrm{SiO}_{2}$ acquired by changing the speed of shave-off scan. These profiles were normalized at the maximum intensity of each profile. In our previous study, we proposed the method to determine the value of $\alpha$ from acquired shave-off depth profile $[4,5]$. We estimated the value of $\alpha$ of each acquired shave-off depth profile and calculated the 'shave-off sputtered atom yield' by using the estimated value of $\alpha$ and the sputtered sample volume.
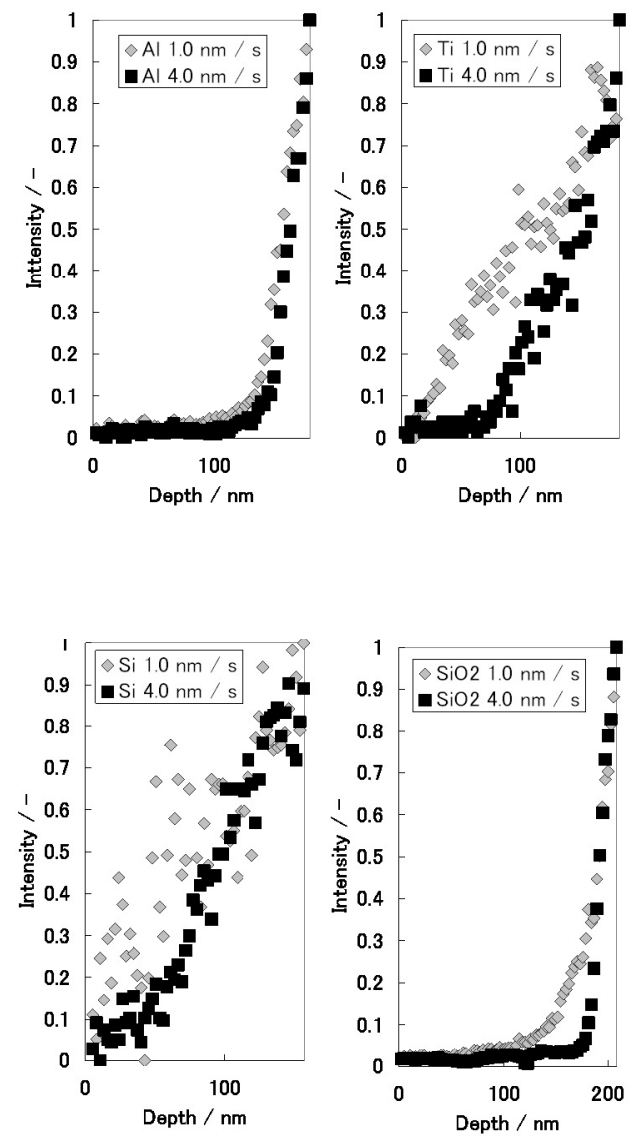

Fig. 5. The shave-off depth profiles of each sample acquired by changing the speed of shave-off scan.

Table 1. The estimated depth resolution and 'shave-off sputtered atom yield' of each sample.

\begin{tabular}{|c|c|c|c|c|}
\hline Target ion & $\mathrm{Al}^{+}$ & $\mathrm{Ti}^{+}$ & $\mathrm{Si}^{+}$ & $\mathrm{Si}^{+}$ \\
\hline Composition of sample & $\mathrm{Al}$ & $\mathrm{Ti}$ & $\mathrm{Si}$ & $\mathrm{SiO}_{2}$ \\
\hline $\begin{array}{c}\text { Depth resolution of } \\
1.0 \mathrm{~nm} / \mathrm{s} \text { [nm] }\end{array}$ & 29 & 85 & 66 & 48 \\
\hline $\begin{array}{c}\text { Depth resolution of } \\
4.0 \mathrm{~nm} / \mathrm{s} \text { [nm] }\end{array}$ & 24 & 59 & 51 & 34 \\
\hline $\begin{array}{c}\text { Shave-off sputtered } \\
\text { atom yield [atoms/ion] }\end{array}$ & 3.6 & 21 & 7.2 & 5.4 \\
\hline
\end{tabular}

Table 1 shows the estimated depth resolution and 'shave-off sputtered atom yield'. The changing speed of shave-off scan toward the depth direction is essentially the same meaning with the changing the thickness of sample. In other words, the depth resolution becomes worse when the sample becomes thick. On the other hand, the sample is thinned down, the acquirable secondary ions were reduced and $\mathrm{S} / \mathrm{N}$ changes worse. For these reasons, thinning the sample is restricted. In addition, the scanning speed is also restricted by the control system. Thus, the optimized condition should be determined for the both sides of the thickness of sample and the scanning speed. Moreover, it was emerged that each sample has different 'shave-off sputtered atom yield' even between $\mathrm{Si}^{+}$in $\mathrm{Si}$ and $\mathrm{Si}^{+}$in $\mathrm{SiO}_{2}$.

These results suggest that the optimized experimental condition is needed to be determined for every measurement to achieve the ultimate depth resolution.

\section{Conclusion}

We evaluated the influence of the long tail of the FIB in detail using new simulation method to clarify the prospective between the FIB and the sample in the shave-off condition. As a result, it was suggested that each sample has different 'shave-off sputtered atom yield' and the optimized experimental conditions can be determined by the simulation method we proposed for the achievement of ultimate depth resolution.

\section{Acknowledgement}

This work was supported in part by Global COE program "Chemistry Innovation through Cooperation of Science and Engineering", MEXT, Japan.

\section{References}

[1] M. Toi, A. Maekawa, T. Yamamoto, B. Tomiyasu, T. Sakamoto, M. Owari, M. Nojima and Y. Nihei, J. Surf. Anal., 12, 170 (2005).

[2] W. Ward, R. L. Kubena, M. W. Utlaut, J. Vac. Sci. Technol., B6, 2090 (1988).

[3] K. Nakamura, Y. Ishikawa, K. Utsumi, H. Igusa, R. Tanaka, Y. Ishizaki, T. Yamamoto, A. Maekawa, M. Owari, M. Nojima and Y. Nihei, Surf. Interf. Anal. 38, 1734 (2006).

[4] M. Fujii, K. Nakamura, Y. Ishizaki, M. Nojima, M. Owari and Y. Nihei, Applied Surface Science, 255, 1354, (2008).

[5] M. Fujii, M. Nojima, M. Owari and Y. Nihei, e-Journal of Surface and Nanotechnology, 8, 247 
Journal of Surface Analysis Vol.17 No.3 (2011) pp.232-236

M.Fujii et al. Evaluation of Focused Ion Beam for Shave-off Depth Profiling

(2010).

[6] F.A. Stevie, C. B. Vartuli, L. A. Giannuzzi, T. L.

Shofner, S. R. Brown, B. Rossie, F. Hillion, R. H.

Mills, M. Antonell, R. B. Irwin and B. M. Purcell,

Surf. Interface Anal., 31, 345 (2001). 